\title{
Preparation of Fine Particles with Improved Solubility Using a Complex Fluidized-Bed Granulator Equipped with a Particle-Sizing Mechanism
}

\author{
Shouichi Hosaka,* Yasufumi Okamura, and Yuji Tokunaga \\ Research and Development Division, Sawai Pharmaceutical Co., Ltd.; 5-2-30 Miyahara, Yodogawa-ku, Osaka 532- \\ 0003, Japan.
}

Received August 17, 2015; accepted March 2, 2016

\begin{abstract}
A new type of fluidized-bed granulator equipped with a particle-sizing mechanism was used for the preparation of fine particles that improved the solubility of a poorly water-soluble drug substance. Cefteram pivoxyl (CEF) was selected as a model drug substance, and its solution with a hydrophilic polymer, hydroxypropyl cellulose (HPC-L), was sprayed on granulation grade lactose monohydrate (Lac). Three types of treated particles were prepared under different conditions focused on the spraying air pressure and the amount of HPC-L. When the amount of HPC-L was changed, the size of the obtained particles was similar. However, particle size distribution was dependent on the amount of HPC-L. Its distribution became more homogenous with greater amounts of HPC-L, but the particle size distribution obtained by decreasing the spraying air pressure was not acceptable. By processing CEF with HPC-L using a complex fluidized-bed granulator equipped with a particle-sizing mechanism, the dissolution ratio was elevated by approximately $40 \%$ compared to that of unprocessed CEF. Moreover, in the dissolution profile of treated CEF, the initial burst was suppressed, and nearly zero order release was observed up to approximately $60 \%$ in the dissolution profile. This technique may represent a method with which to design fine particles of approximately $100 \mu \mathrm{m}$ in size with a narrow distribution, which can improve the solubility of a drug substance with low solubility.
\end{abstract}

Key words fine particle; particle size distribution; dissolution; complex fluidized-bed granulator

Recently, techniques that can increase the dissolution ratio or its rate have become a focus of attention since many drugs with low water-solubility are being developed. One method to improve these drugs is to treat drug substances with hydrophilic polymers. The spray-drying technique is a well-known method for the improvement of solubility. ${ }^{1)}$ The advantage of this method is that the particle size of the powder obtained can be controlled in a single step. ${ }^{2}$ However, pharmaceutical companies encounter several problems, such as the need for new equipment (especially at a commercial scale) and patent or license problems. On the other hand, the approach of spraying a solution or dispersion containing drug substance and hydrophilic polymer on cellulose-based beads using a rotating fluidized-bed granulator is a more practical method. In this case, considering the size of cellulose-based beads, the particle size obtained would be over $200 \mu \mathrm{m}$ so that the size of tablets containing them tends to be large. ${ }^{3)}$ To obtain fine particles of approximately $100 \mu \mathrm{m}$ in size, reduction in the size of the core particle is needed. At this time, however, the production of agglomerate particles may be a problem when using a conventional rotating fluidized-bed granulator. ${ }^{4)}$ To suppress their production, a new type of fluidized-bed granulator equipped with a particle-sizing mechanism, referred to as a complex fluidized-bed granulator, has been developed. ${ }^{5)}$ Few studies in the pharmaceutical field have been reported concerning the improvement of solubility of a poorly watersoluble drug substance using this device.

The goal of this study was to design fine particles of approximately $100 \mu \mathrm{m}$ in size with a narrow distribution, which improved the solubility of drug substance. The reason for setting target particle size is as follows. 1) To ensure good flowability of treated particles considering direct compression or capsule filling process. 2) To expect good content uniformity when tablets or capsules are prepared using this particles (homogenous particle size distribution is desirable). 3) To expect reduction of tablet size by decreasing treated particle size and so on.

Cefteram pivoxyl (CEF) was selected as a model drug substance with low water-solubility. Granulation grade lactose monohydrate (Lac) was selected as a core particle. A solution of CEF containing a hydrophilic polymer, hydroxypropyl cellulose (HPC-L) was sprayed on Lac particles using a complex fluidized-bed granulator. In the present study, to explore critical factors focused on particle size and its distribution, the effect of the spraying air pressure and the amount of HPC-L is investigated under two conditions. Moreover, dissolution profile from treated CEF with a suitable particle size distribution was investigated.

\section{Experimental}

Materials Cefteram pivoxyl (JEIL Pharmaceutical Co., Ltd., South Korea) was used as a low water-solubility drug substance. Lactose monohydrate (Pharmatose ${ }^{\circledR}$ 200M, DMV, The Netherlands) and hydroxypropyl cellulose (HPC-L, Nihon Soda Co., Ltd., Japan) were used as the core particle and hydrophilic polymer, respectively. Both excipients are listed in the Japanese Pharmacopoeia (JP).

Preparation of Treated CEF Three types of treated CEF, which differed in the spraying air pressure and the amount of HPC-L, were prepared in a complex fluidized-bed granulator equipped with a particle-sizing mechanism (SFP-01, Powrex Corporation, Japan). The schematic representation of a complex fluidized-bed granulator is shown in Fig. 1.

The formulations of the three types of treated particles, CEF-1, CEF-1a and CEF-2, are listed in Table 1. The concentration of spraying solution (ratio of solid components to 


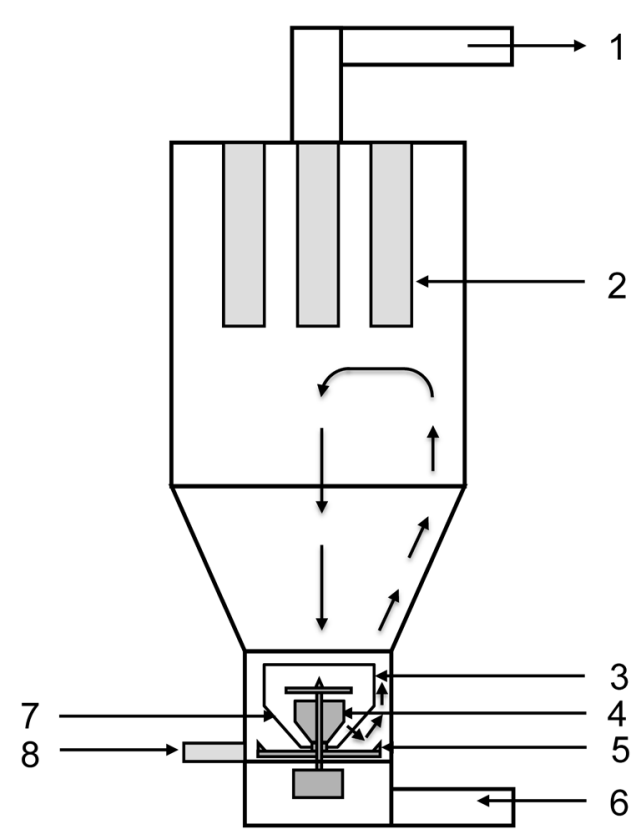

Fig. 1. Schematic Representation of a Complex Fluidized-Bed Granulator

1: Exhaust air, 2: bag filter, 3: partition tube, 4: impeller, 5: rotor disc, 6: inlet air, 7: screen, 8: spray nozzle.

Table 1. Formulations of Treated Particles, CEF-1, CEF-1a and CEF-2, Prepared Using a Complex Fluidized-Bed Granulator

\begin{tabular}{lcr}
\hline \hline \multirow{2}{*}{ Formulations } & \multicolumn{2}{c}{ Amount $(\mathrm{g})$} \\
\cline { 2 - 3 } & CEF-1, CEF-1a & CEF-2 \\
\hline Core particle & & \\
Lactose monohydrate & 400.0 & 400.0 \\
Spraying solution & & \\
Cefteram pivoxyl & 80.0 & 80.0 \\
Hydroxypropyl cellulose & 4.0 & 16.0 \\
Ethanol & 444.8 & 531.2 \\
Purified water & 7.2 & 28.8 \\
\hline Total & 936.0 & 1056.0 \\
\hline
\end{tabular}

total amount) was set at $15 \%$ considering both the viscosity of spraying solution and the spraying time. At the higher concentration, spraying troubles would happen due to the increased viscosity of spraying solution. At the lower concentration, spraying time would be prolonged. The operating conditions discussed in this study are relatively practical considering industrialization.

Lac (400g) was fed into the device and spraying solutions containing the equivalent of $80 \mathrm{~g}$ of CEF and $4 \mathrm{~g}$ (CEF-1), or $16 \mathrm{~g}$ (CEF-2) of HPC-L were prepared. The screen diameter in this device was $2.0 \mathrm{~mm} \phi$ and the impeller in the partition tube, which rotated together with the rotor disc was set at $1000 \mathrm{rpm}$. The operating inlet airflow was set at $30 \mathrm{~m}^{3} / \mathrm{h}$ taking into consideration powder mobility and its temperature was set at $60^{\circ} \mathrm{C}$. The spray nozzle was placed on the bottom of the apparatus. The spraying air pressure and spraying rate were set at $0.16 \mathrm{MPa}$ and $4.5 \mathrm{~g} / \mathrm{min}$, respectively. After spraying, the particles were dried at $60^{\circ} \mathrm{C}$ for $5 \mathrm{~min}$ in the same apparatus. CEF-1a was prepared for the same formulation and operating condition as CEF-1 except for the spraying air pressure, which was set at $0.12 \mathrm{MPa}$.

Observation of Treated CEF The appearance of CEF-1, CEF-1a, CEF-2 and Lac was examined using digital microscope (VHX-1000, KEYENCE, Japan). Thirty particles of CEF-1, CEF-1a and CEF-2 were used for the particle size measurement. The particle size was expressed as a mean diameter together with a standard deviation. Glass beads (GBL-100, The Association of Powder Process Industry and Engineering, Japan) were used as a reference.

The surface morphology of CEF-1, CEF-1a, CEF-2 and Lac was also examined using scanning electron microscopy (SEM) (JCM-5100, JEOL, Japan) with accelerating voltage of $20 \mathrm{kV}$ and magnification of $\times 500$. Prior to the observation, the samples were sputter coated (E-1010, Hitachi, Japan) with a gold layer under a vacuum for $30 \mathrm{~s}$.

Particle Size Distribution of Treated CEF The particle size distribution of CEF-1, CEF-1a and CEF-2 was examined using a laser diffraction method (LS 13 320, Beckman Coulter, Inc., U.S.A.).

Assay in Treated CEF Prior to the dissolution testing, the assay of CEF-1 and CEF-2 was investigated using the HPLC (LC-10A, Shimadzu Corporation, Japan) system mentioned below. Treated particles equivalent to $20 \mathrm{mg}$ of CEF were dissolved in $50 \mathrm{~mL}$ of a mixture of water-acetonitrile $(1: 1, \mathrm{v} / \mathrm{v})$ and filtered $(0.45 \mu \mathrm{m})$ as a sample for HPLC. The mobile phase consisted of acetic acid and sodium acetic acid buffer solution (adjusted to $\mathrm{pH} 5.0)$-acetonitrile $(62: 38, \mathrm{v} / \mathrm{v})$, which flowed through the column (Inertsil ODS-2, $150 \mathrm{~mm} \times 4.6 \mathrm{~mm}$ i.d., GL Sciences, Japan) at a flow rate of $1.0 \mathrm{~mL} / \mathrm{min}$. The mobile phase was degassed before use. The column was maintained at $40^{\circ} \mathrm{C}$. Measurements were made with an injection volume of $10 \mu \mathrm{L}$ and ultraviolet (UV) detection at $254 \mathrm{~nm}$. The assay in treated CEF was expressed as the average of three samples.

Drug Release from Unprocessed and Treated CEF Dissolution testing was performed using the JPXIV paddle method (Dissolution Tester NTR-6100A, Toyama Sangyo Co., Ltd., Japan), which was equivalent to $20 \mathrm{mg}$ of $\mathrm{CEF}$ in both CEF-1 and CEF-2 at $37 \pm 0.5^{\circ} \mathrm{C}$ in $900 \mathrm{~mL}$ of water. The amount of the sample for the dissolution testing was corrected by the assay. The medium was degassed before use and the paddle rotation speed was set at $100 \mathrm{rpm}$. Each $10 \mathrm{~mL}$ sample was withdrawn from the dissolution vessels at appropriate intervals and an equal volume of fresh medium immediately added. Samples were then passed through a membrane filter. The quantity of CEF was assayed with a UV spectrophotometer (UV/VIS Spectrophotometer, V-530, Jasco Corporation, Japan) at $300 \mathrm{~nm}$. The dissolution ratio of unprocessed and treated CEF was plotted as the average of two samples with maximum and minimum range.

Crystallinity of CEF The crystallinity of CEF was examined using a powder X-ray diffractometer (RINT 2100VK, Rigaku, Japan) with target of $\mathrm{Cu} K \alpha$, voltage of $40 \mathrm{kV}$, current of $40 \mathrm{~mA}$, scan speed of $2 \% \mathrm{~min}$ and $2 \theta$ range from 2 to 40 .

Interaction between CEF and HPC-L The possibility of the interaction between $\mathrm{CEF}$ and HPC-L was examined using a Fourier transform infrared (FT-IR) spectrophotometer (FT-IR-8200PC, Shimadzu Corporation, Japan) with potassium bromide disk method. Polystyrene film was used as a reference. 


\section{Results and Discussion}

Powder Properties of Treated CEF In general, cellulose-based beads like microcrystalline cellulose spheres $\left(\right.$ Celphere $\left.{ }^{\circledR}\right)$ or lactose monohydrate-microcrystalline cellulose spheres $\left(\right.$ Nonpareil $\left.{ }^{\circledR}\right)$ are selected as a core particle for coating in a rotating fluidized-bed granulator., ${ }^{6,7)}$ In such cases, the particles after coating would be over $200 \mu \mathrm{m}$ in size considering the size of the core particle. To obtain fine particles of approximately $100 \mu \mathrm{m}$ in size, which would improve the solubility of the drug substance, reduction of the size of the core particle is needed. In the present study, granulation grade Lac was selected as the core particle with a diameter of $56 \mu \mathrm{m}$. The first choice for improving solubility is to treat the drug substance with a hydrophilic polymer. HPC-L, which is conventionally used as a binder was selected as a hydrophilic polymer.

A new type of fluidized-bed granulator equipped with a particle-sizing mechanism to prevent the production of agglomerate was used. The coating solution of CEF with HPC-L is sprayed on Lac particles and the composite particles are dried during the upturn along the wall of the container by the rotating airflow. The composite particles then fall into a partition tube and are dispersed by an impeller rotating at a high speed. The dispersed particles pass through a screen and the coating solution is sprayed again. This cycle is repeated during the processing until the solution for spraying is depleted. Due to the rotating impeller in this device, the production of agglomerate is suppressed. Several factors, such as inlet airflow rate and spraying rate as well as concentration of spraying solution were optimized for the operation.

In the present study, the spraying air pressure and the amount of HPC-L is investigated under two conditions as critical factors, which may influence to the particle size and its distribution. The spraying air pressure was set at 0.16 and $0.12 \mathrm{MPa}$ in this experiment and compared particle size and its distribution between them. Actually, another level of the spraying air pressure for example, $0.20 \mathrm{MPa}$ or more should be investigated. However, at the higher spraying air pressure: $0.20 \mathrm{MPa}$ or more, solvent of spraying solution tends to evaporate rapidly and spraying solution becomes dust before attaching to Lac particles, especially in the ethanol based solution. Accordingly, two suitable levels of the spraying air pressure are selected in this experiment. The amount of HPC-L was set at 4 and $16 \mathrm{~g}$ in this experiment. Actually, another level of the amount of HPC-L for example, $24 \mathrm{~g}$ or more should be investigated. However, at this time, spraying time prolongs dramatically and it is not suitable for practical industrialization. Accordingly, two suitable levels of the amount of HPC-L are selected in this experiment.

The appearance and surface morphology of CEF-1, CEF-1a, CEF-2 and Lac are shown in Figs. 2 and 3, respectively. The state of raw material, Lac particles was agglomerate. After treating, agglomerate particles in Lac disappeared and treated CEF appeared to be coated on Lac particles. Moreover, various particles in size, especially agglomerate were observed in CEF-1a and the particle size of CEF-2 seemed to be more homogenous than the other treated CEF.

Particle size distribution measured by a laser diffraction method is shown in Fig. 4. In CEF-1, fraction of dust was most observed (arrow in left side of Fig. 4) and in CEF-1a, fraction of agglomerate was also observed as mentioned above (arrow in center of Fig. 4). In CEF-2, particle size distribution was more homogenous than the other treated $\mathrm{CEF}$, which was consistent with the result of digital microscope observation.

From the digital microscope analysis, the particle size, together with standard deviation of treated CEF are listed in Table 2. Particle size of CEF-1 was $90 \mu \mathrm{m}$ as we expected. Furthermore, we investigated whether its distribution (standard deviation) could be improved. The effect of the spraying air pressure on particle size and its distribution was investigated. However, particle size and its distribution of CEF-1a were greater than those of CEF-1, which should be more improved. The effect of the amount of HPC-L on particle size and its distribution was investigated. Particle size of CEF-2 was the
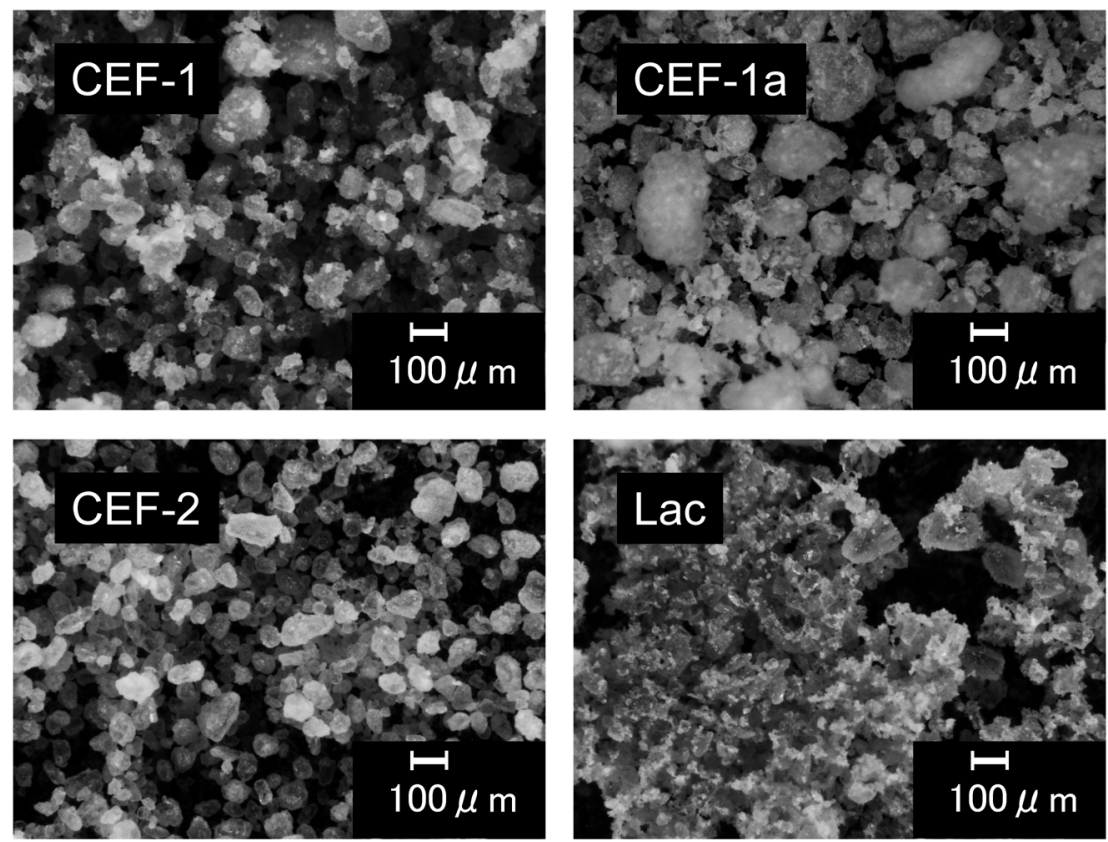

Fig. 2. Digital Microscope Image of Lac and Treated Particles, CEF-1, CEF-1a, and CEF-2, Prepared Using a Complex Fluidized-Bed Granulator 

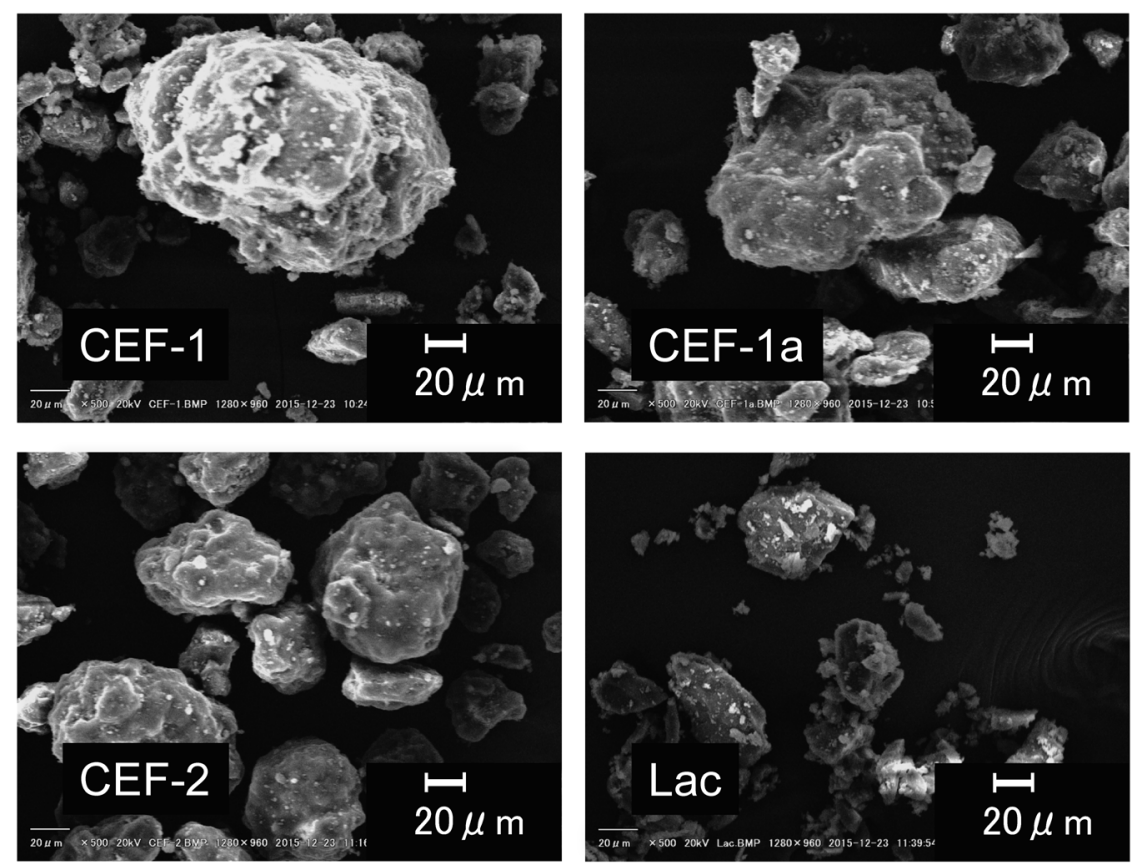

Fig. 3. Scanning Electron Microscopy (SEM) of Lac and Treated Particles, CEF-1, CEF-1a, and CEF-2, Prepared Using a Complex Fluidized-Bed Granulator
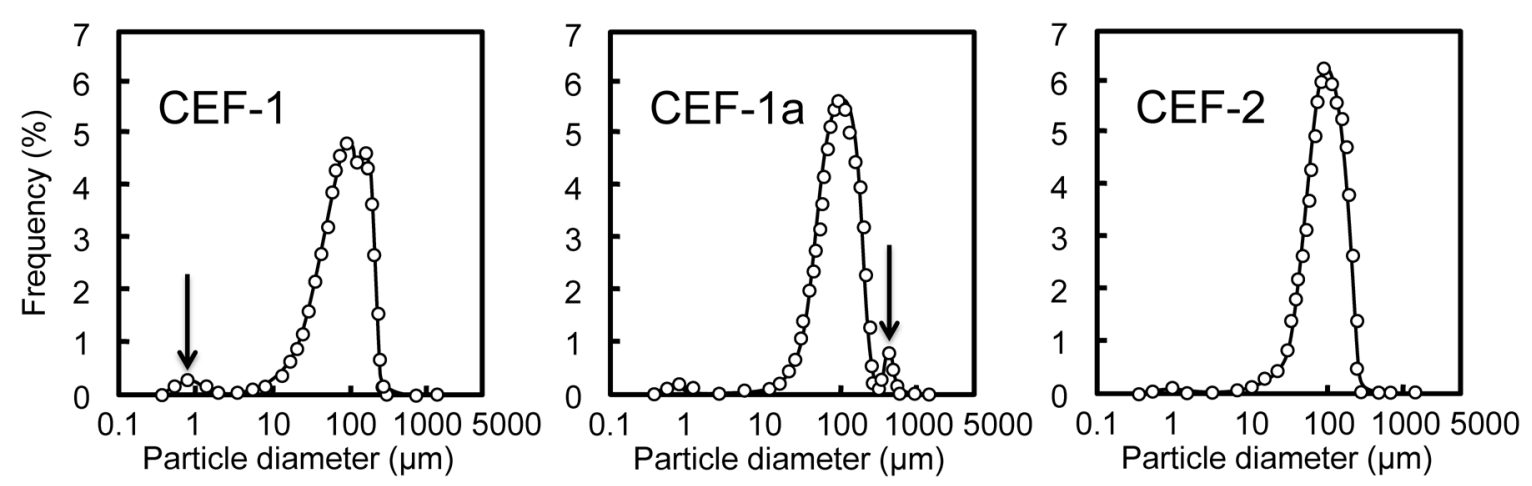

Fig. 4. Particle Size Distributions of Treated Particles, CEF-1 CEF-1a and CEF-2, Prepared Using a Complex Fluidized-Bed Granulator

Table 2. Particle Size and Its Distribution (Standard Deviation) of Treated Particles, CEF-1 CEF-1a and CEF-2, Prepared Using a Complex Fluidized-Bed Granulator

\begin{tabular}{cccc}
\hline \hline Treated CEF & CEF-1 & CEF-1a & CEF-2 \\
\hline Particle size $(\mu \mathrm{m})$ & 89.7 & 124.6 & 85.2 \\
Standard deviation & 54.8 & 82.2 & 30.7 \\
\hline
\end{tabular}

same extent as that of CEF-1, which was $85 \mu \mathrm{m}$ and its distribution of CEF-2 was most homogenous.

It is suggested that spraying mist size increases as the spraying air pressure decreases from 0.16 to $0.12 \mathrm{MPa}$. This is the reason for the observation of agglomerate in CEF-1a. On the other hand, the reason for the observation of dust is due to the higher spraying air pressure and insufficient amount of binder. At this time, the composite of CEF and HPC-L are produced by rapid evaporation of solvent before attaching to Lac particles. In CEF-2, fraction of dust decreased compared to that in CEF-1. It is suggested that in CEF-2, the amount of HPC-L, which acts as a binder, is sufficient so that fraction of dust may decrease.
Prior to the dissolution testing, the assay of CEF-1 and -2 was investigated. The assay of CEF-1 and -2 was 93.0 and $97.9 \%$, respectively. The assay of CEF-2 was greater than that of CEF-1. One of the reasons for this is suggested as follows. In CEF-1, dust is more produced due to the higher spraying air pressure and insufficient amount of binder so that a part of dust may pass through bag filter with exhaust air. Because dust may be the composite of CEF and HPC-L, loss of assay would be observed. This phenomenon is sometimes observed in operating conventional fluidized-bed device. From the points of view of particle size distribution and assay, CEF-2 is preferable. For preparing particles with a narrow distribution, it was more effective to increase the amount of HPC-L than to decrease the spraying air pressure.

Drug Release Profile of Unprocessed and Treated CEF The amount of the samples for the dissolution testing was corrected by the assay as mentioned in the previous section. Drug release profiles for unprocessed and treated CEF are shown in Fig. 5. The dissolution ratio of unprocessed CEF increased as a function of time and was approximately $45 \%$ at $60 \mathrm{~min}$. In the case of CEF-1, the dissolution ratio increased as a function 
of time and was approximately $85 \%$ at $60 \mathrm{~min}$. A similar profile was obtained for CEF-2. By processing CEF with HPC-L using a complex fluidized-bed granulator, the final dissolution ratio was elevated by about $40 \%$ compared to that of unprocessed one.

Here, we focused on the dissolution profiles of CEF-1 and -2 at the initial stage. In general, the dissolution profile of drug substance from particles is rapid. In CEF-1 and -2 , it was noted that the initial burst was suppressed and nearly zero order release was observed up to around $60 \%$ and these are believed to be unique characteristics. After that the dissolution rate was retarded above $60 \%$ because saturated state of $\mathrm{CEF}$ might be approached.

Characterization of Unprocessed and Treated CEF In general, to improve the dissolution ratio or its rate, reduction of particle size or decrease of crystallinity of drug substance is often performed. However, the particle size of unprocessed CEF was $3 \mu \mathrm{m}$, which was too small to pulverize more and the crystal state of unprocessed CEF was amorphous by powder X-ray diffraction pattern as shown in Fig. 6(A). It is strongly suggested that the reason for the elevation of the dissolution ratio in treated $\mathrm{CEF}$ is not reduction of particle size or decrease of crystallinity. The elevation of the dissolution ratio is believed to be due to improvement of the wettability of drug substance by treatment with a hydrophilic polymer.

In the drug release profile, nearly zero order release was observed at the initial stage. The reason for the characteristic dissolution profile may be due to the interaction between drug substance and polymer via hydrogen bonding. Several spray-dried drug substances with hydrophilic polymers form solid dispersions with the interaction between drug substances and polymers. ${ }^{8-10)}$ A solid dispersion via hydrogen bonding is formed when a solution of cefditoren pivoxyl, which is a $\beta$-lactam antibiotic that has a structure similar to that of $\mathrm{CEF}$, is spray-dried together with the hydrophilic polymer, hydroxypropyl methylcellulose or HPC-L. ${ }^{10)}$ Furthermore, in our another experiment as a reference, the solution of CEF with hydroxypropyl methylcellulose was sprayed using a conventional fluidized-bed granulator and composite particles were prepared. FT-IR spectrum of the composite particles compared with its physical mixture is shown in Fig. 6(B). The peaks of 3340 and $2978 \mathrm{~cm}^{-1}$ in physical mixture were shifted to 3337 and $2975 \mathrm{~cm}^{-1}$ in composite particles, respectively. It is suggested that the interaction is formed between CEF and hydroxypropyl methylcellulose. Accordingly, in the present study, the interaction may be formed via hydrogen bonding between $\mathrm{CEF}$ and HPC-L as the cases mentioned above.

Here, considering the dissolution mechanism from CEF-1 and -2 particles, drug substance must overcome an energy barrier corresponding to the interaction between the drug substance and the polymer. ${ }^{11)}$ Agrawal et al. reported that the breakage of the drug-polymer interaction may be a much slower step as compared to the diffusion of drug substance through the polymer network ${ }^{11)}$ so nearly zero order release may be observed up to approximately $60 \%$ in the dissolution profile.
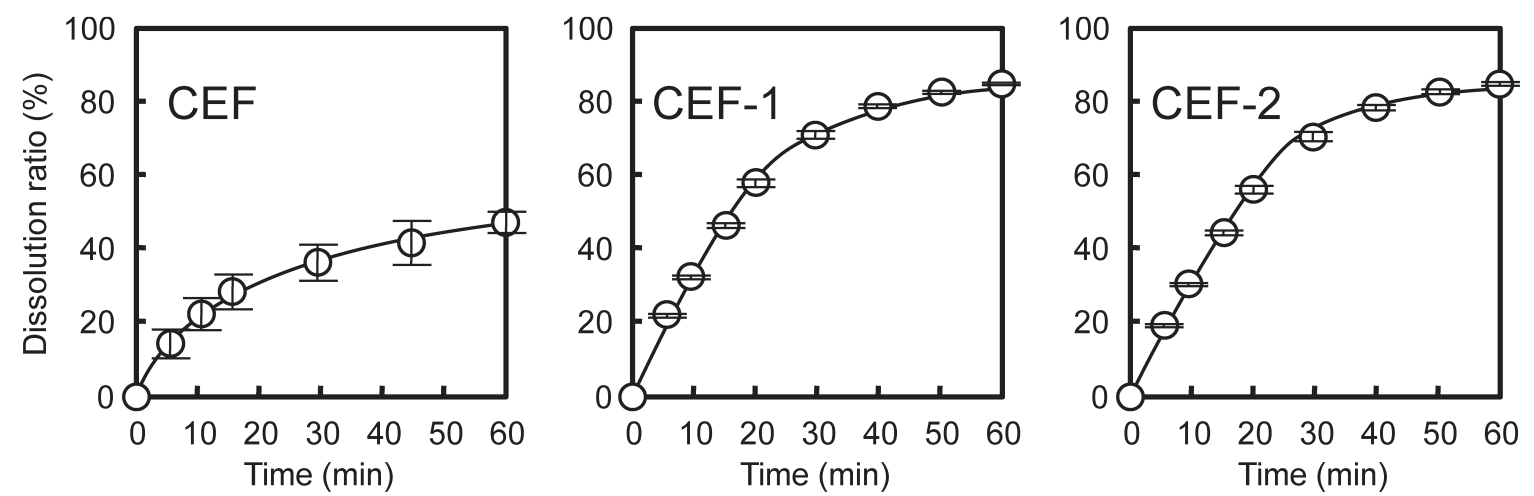

Fig. 5. Dissolution Profile of Unprocessed CEF and Treated Particles, CEF-1 and CEF-2, Prepared Using a Complex Fluidized-Bed Granulator
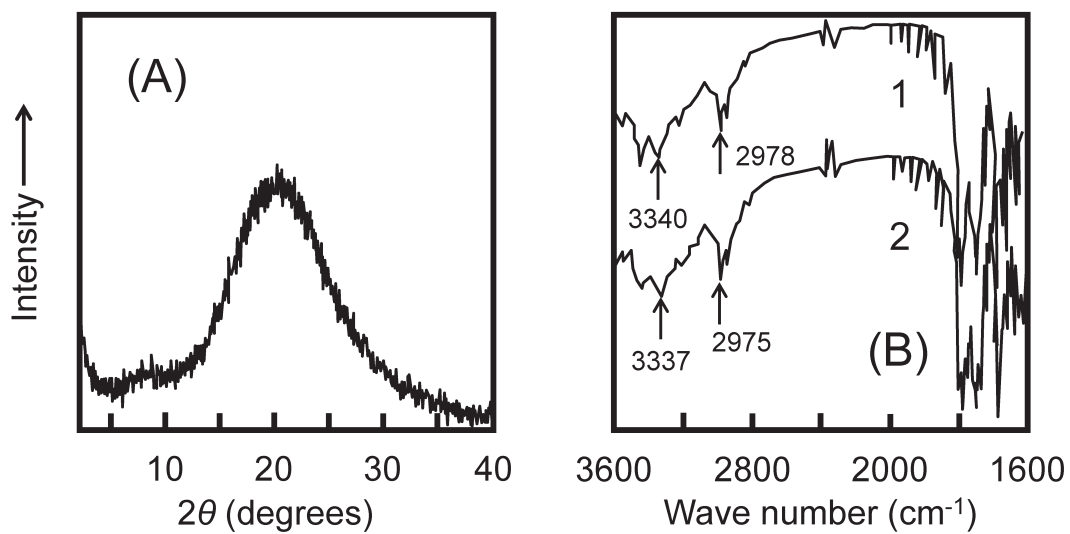

Fig. 6. Physicochemical Property of Unprocessed CEF and Composite Particles Consist of CEF and Hydroxypropyl Methylcellulose, Prepared Using a Conventional Fluidized-Bed Granulator

(A) Powder X-ray diffraction pattern of unprocessed CEF, (B) FT-IR spectrums of physical mixture and composite particles. 1: Physical mixture, 2: composite particles. 
In the present study, particles with a diameter of approximately $100 \mu \mathrm{m}$, which improved solubility, could be obtained readily using a complex fluidized-bed granulator equipped with a particle-sizing mechanism. Moreover, the particle size distribution could be improved by considering the amount of HPC-L added without changing the dissolution profiles. In the dissolution profile from these particles, an initial burst was suppressed. These findings are significant and applicable to the design of fine particles of approximately $100 \mu \mathrm{m}$ that have the desired dissolution profiles.

\section{Conclusion}

Three types of treated particles were prepared using a complex fluidized-bed granulator equipped with a particle-sizing mechanism under different conditions focused on the spraying air pressure and the amount of HPC-L. When the amount of HPC-L was changed, the size of the obtained particles was similar. However, particle size distribution was dependent on the amount of HPC-L. Its distribution became more homogenous with greater amounts of HPC-L, but the particle size distribution obtained by decreasing the spraying air pressure was not acceptable.

By processing CEF with HPC-L using this device, the dissolution ratio was elevated by approximately $40 \%$ compared to that of unprocessed one. Moreover, in the dissolution profile of treated CEF, the initial burst was suppressed, and nearly zero order release was observed up to approximately $60 \%$ in the dissolution profile.
The present method is applicable to the design of fine particles of approximately $100 \mu \mathrm{m}$ in size with a narrow distribution, which improved the solubility of drug substance.

Conflict of Interest The authors declare no conflict of interest.

\section{References}

1) Yin S. X., Franchini M., Chen J., Hsieh A., Jen S., Lee T., Hussain M., Smith R., J. Pharm. Sci., 94, 1598-1607 (2005).

2) Elversson J., Millqvist-Fureby A., J. Pharm. Sci., 94, 2049-2060 (2005).

3) Ho H.-O., Su H.-L., Tsai T., Sheu M.-T., Int. J. Pharm., 139, $223-$ 229 (1996).

4) Kimura S., Iwao Y., Ishida M., Uchimoto T., Miyagishima A., Sonobe T., Itai S., Int. J. Pharm., 391, 244-247 (2010).

5) Terashita K., Natsuyama S., Nagato T., Kanou Y., Namba N., Pharm. Tech. Jpn., 20, 105-113 (2004).

6) Watano S., Yamamoto A., Miyanami K., Chem. Pharm. Bull., 44, 2128-2131 (1996).

7) Shimizu T., Nakano Y., Morimoto S., Tabata T., Hamaguchi N., Igari Y., Chem. Pharm. Bull., 51, 942-947 (2003).

8) Hirasawa N., Ishise S., Miyata H., Danjo K., Drug Dev. Ind. Pharm., 29, 339-344 (2003).

9) Tantishaiyakul V., Kaewnopparat N., Ingkatawornwong S., Int. J. Pharm., 143, 59-66 (1996).

10) Yokoi Y., Yonemochi E., Terada K., Int. J. Pharm., 280, 67-75 (2004)

11) Agrawal S. K., Sanabria-DeLong N., Coburn J. M., Tew G. N., Bhatia S. R., J. Control. Release, 112, 64-71 (2006). 